POS PROCEEDINGS

\title{
$W^{\prime}$ and $Z^{\prime}$ searches at the LHC
}

\section{Elena ACCOMANDO**}

School of Physics \& Astronomy, University of Southampton, Southampton SO17 1BJ, UK

E-mail: e.accomando@soton.ac.uk

\section{Diego BECCIOLINI}

$C P^{3}$-Origins \& the Danish Institute for Advanced Study DIAS, University of Southern Denmark, Campusvej 55, DK-5230 Odense, Denmark

E-mail: beccioliniecp3.dias.sdu.dk

\section{Alexander BELYAEV}

School of Physics \& Astronomy, University of Southampton, Southampton SO17 1BJ, UK

E-mail: A.Belyaev@soton.ac.uk

\section{Stefania DE CURTIS}

I.N.F.N. Sezione di Firenze, via G. Sansone 1, 50019 Sesto Fiorentino, Italy

E-mail: decurtisefi.infn.it

\section{Daniele DOMINICI}

Universitá di Firenze, Dip. Fisica e Astronomia, via G. Sansone 1, 50019 Sesto Fiorentino, Italy

E-mail: dominiciefi.infn.it

\section{Steve F. KING}

School of Physics \& Astronomy, University of Southampton, Southampton SO17 1BJ, UK

E-mail: S.F.King@soton.ac.uk

\section{Stefano MORETTI}

School of Physics \& Astronomy, University of Southampton, Southampton SO17 1BJ, UK

E-mail: s.moretti@soton.ac.uk

\section{Claire SHEPHERD-THEMISTOCLEOUS}

PPD, Rutherford Appleton Laboratory, Chilton, Didcot, Oxon OX11 OQX, UK

E-mail: Claire.Shepherdastfc.ac.uk

Searches for extra heavy $W^{\prime}$ and $Z^{\prime}$-bosons in the leptonic Drell-Yan channel at the Large Hadron Collider (LHC) are favoured by present and future data. We focus on a common approximation used in theoretical and experimental analyses: neglecting interference between the new gauge bosons $W^{\prime \pm}\left(Z^{\prime}\right)$ and the Standard Model ones $W^{ \pm}(Z, \gamma)$. And present the implications of adopting this approximation on the data interpretation and the extraction of exclusion limits on $W^{\prime}$ and $Z^{\prime}$ boson masses. First results from experimental analyses are quoted.

XXI International Workshop on Deep-Inelastic Scattering and Related Subjects 22-26 April, 2013

Marseilles, France

\footnotetext{
* Speaker.

${ }^{\dagger}$ The speaker is very grateful to the Organizing committee for the invitation and hospitality. This research is supported in part through the NExT Institute.
} 


\section{Introduction}

Drell-Yan (DY) processes, giving rise to the production of lepton pairs in the final state, are very powerful for discovering or bounding new physics involving heavy spin-1 gauge bosons. Scenarios predicting such exotic particles are particularly relevant now as the Large Hadron Collider (LHC) has already collected a generous number of events, and will be soon probing higher energies. We thus address the search for extra $W^{\prime}$ and $Z^{\prime}$ gauge bosons at the LHC, focussing on the common approximations used in the theoretical and experimental analyses, and showing their impact on the data interpretation.

Let us start reviewing the state-of-the-art of calculations and tools for DY processes at the LHC. At Next-to-Leading order (NLO) QCD, mass scale dependent K-factors are often considered via different Monte Carlo Event Generators. Also ElectroWeak (EW) radiative corrections can become sizeable at the $\mathrm{TeV}$ scale we are probing now. They can be of the same order of magnitude of the QCD corrections, depending on the jet veto imposed. Recent implementations (see Ref. [1] and references therein) combine both the NLO EW and QED multiple photon corrections with the "default" NLO and Parton Shower QCD contributions, in order to provide the most accurate result. But despite the effort on NLO computations, and their precious results, still the LO evaluation relies on one main approximation: neglecting interference effects between New Physics and Standard Model (SM), because model dependent and CPU time consuming. We thus concentrate on the Leading Order analysis.

Although the effect of interference between the extra gauge bosons $W^{\prime}$ and $Z^{\prime}$ with their corresponding SM ones, $W$ and $Z, \gamma$ respectively, is commonly discussed in this specific context $[2,3,4,5,6,7]$, one still encounters misleading or incomplete statements regarding how large the effect can be depending on the imposed kinematical cuts. Until now, most experimental searches of new particles in DY channels put interference aside when interpreting the available data $[8,9,10,11,12]$. There is however a growing interest in including this effect in the community, and some of the more recent analyses do discuss the matter [13, 14, 15].

In section 2, we discuss these effects for the $W^{\prime}$-boson search, in section 3 for the $Z^{\prime}$-boson search at the LHC. Our results have been obtained for the LHC at $7 \mathrm{TeV}$ and $8 \mathrm{TeV}$. Conclusions are given at the end.

\section{2. $W^{\prime}$ searches in DY channel at the LHC}

Heavy charged $W^{\prime}$ bosons arise in a number of theories that extend the SM gauge group. The Left-Right symmetric (LR) class of models [16] is an old and popular example. A second class is represented by extra dimensional theories [17], where $W^{\prime}$ bosons emerge as Kaluza-Klein excitations of the SM $W$-boson. All these different $W^{\prime}$ s can be ideally produced in DY channel. However, experimental searches of a generic $W^{\prime}$ particle at the LHC are interpreted in the context of a benchmark scenario inspired by Ref. [18], and named SSM. It just includes one extra charged vector boson with couplings to fermions identical to those of the corresponding SM $W$ and no mixing with the EW SM bosons. In the following we consider the SSM, where the extra $W^{\prime}$ is an heavy replica of the SM $W$ boson and is produced at the LHC in the DY process 

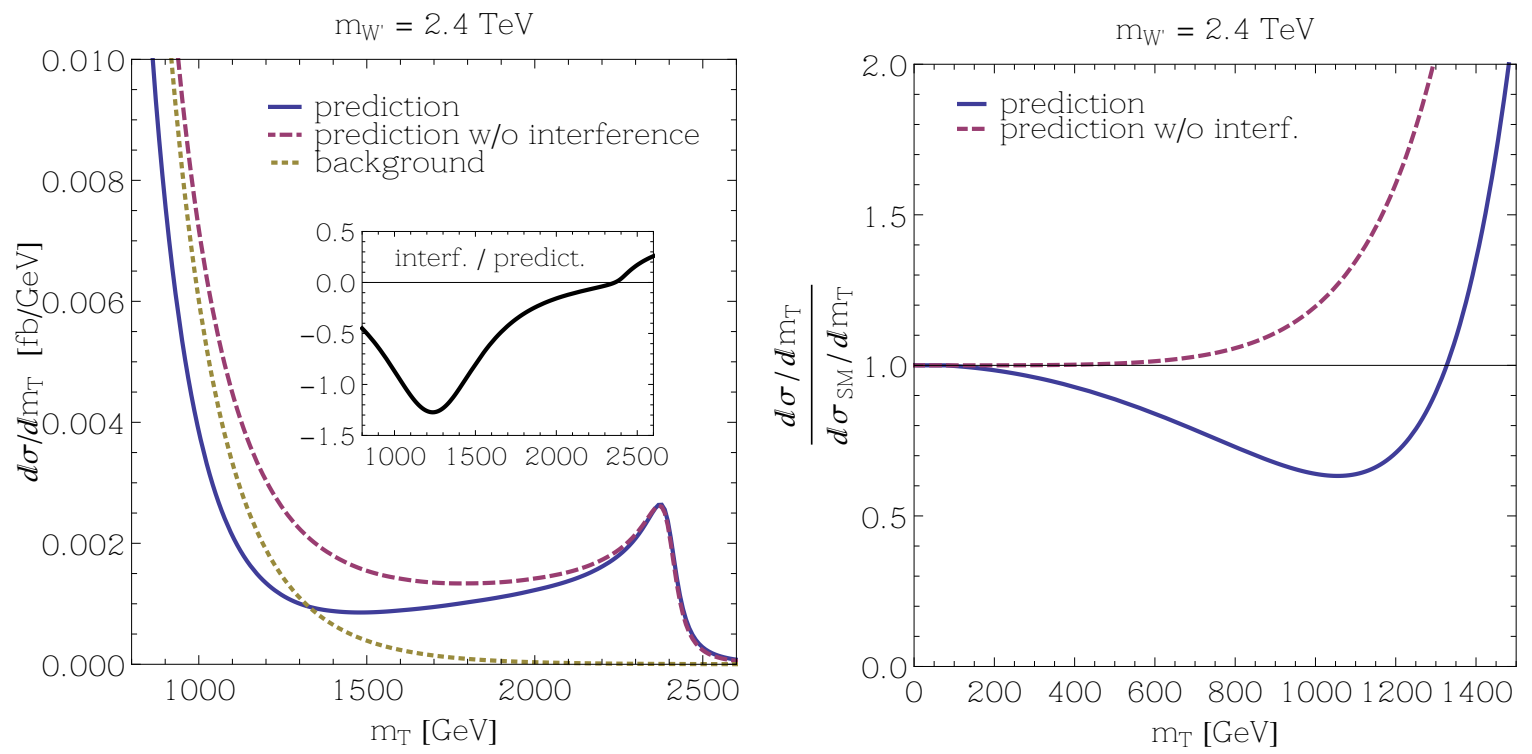

Figure 1: Left: Dilepton transverse mass distribution with and without interfernce, considering $7 \mathrm{TeV} p p$ collisions. The blue solid line shows the complete result with interference, the magenta dashed line without interference. The dotted yellow curve is the SM background. Right: Dilepton transverse mass distribution normalized to the SM one. Color code as in the left plot.

$$
p p \rightarrow W, W^{\prime} \rightarrow l v_{l} \quad l=e, \mu .
$$

The results we briefly summarize in the following are described in detail in Ref. [22].

In Fig.1, we plot the lepton-neutrino transverse mass distribution, and compare the result with (solid line) and without (dashed line) interference between the extra $W^{\prime}$-boson and the SM $W$ boson. The SM background is given as reference (dotted line). Owing to the destructive interference pattern, there is a sizeable reduction of the differential cross section compared to the non-interferred one. The effect is model dependent and can reach up to O(140\%) in the SSM. This generates three main consequences. The first one is that the control region (assumed to be New Physics free) shrinks considerably, as shown in the right plot of Fig. 1 where the ratio between the complete transverse mass distribution and the SM one is given. The second one is that the complete BSM signal might not be positive definite over the full transverse mass range and its shape is model dependent. This is displayed in Fig. 2, where we compare the transverse mass distribution for the complete BSM signal, the pure $W^{\prime}$ signal with no interference and the SM irriducible background as reference. As a consequence, the cumulative BSM signal might not be positive definite. There is a strong dependence on the cut imposed on the transverse mass as shown in the right plot of Fig. 2. The main conclusion is that the unfolded BSM signal cross section, $\sigma(p p \rightarrow l v)-\sigma_{S M}(p p \rightarrow W \rightarrow l v)$ is not a convenient observable anymore, and data should be presented in a different way. Moreover, even if keeping a lower cut on the dilepton transverse mass, the exclusion limits with no interference included are likely to be overestimated. A recent CMS analysis, see the contribution by P. Millet to this conference and Ref. [21], finds that if taking into account interference the bound is $M_{W^{\prime}} \geq$ 3.1 TeV, otherwise if interference is neglected one has $M_{W^{\prime}} \geq 3.35 \mathrm{TeV}$ within the SSM. Starting 

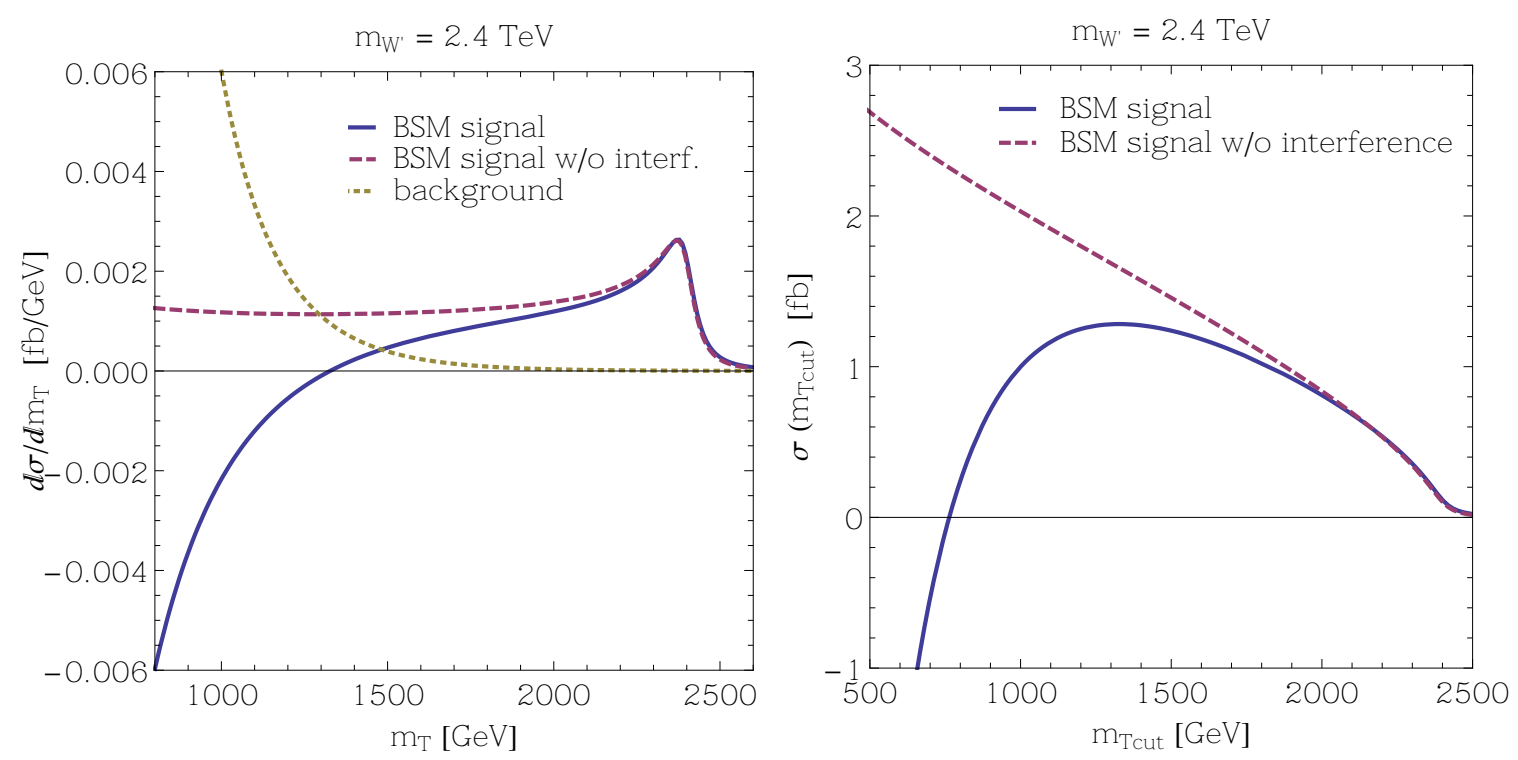

Figure 2: Left: Dilepton transverse mass distribution with and without interfernce. The blue solid line shows the complete BSM signal with interference, the magenta dashed line the pure $W^{\prime}$ signal without interference. The dotted yellow curve is the SM background. Right: Cumulative transverse mass distribution for the BSM signal with (solid blue line) and without (dashed magenta line) interference.

from these results, CMS has elaborated a new way of presenting the 95\% C.L. upper bound on the BSM cross section which accounts for interference effects. And, a $\Delta\left(W^{\prime}\right.$ mass bound $) \simeq 700 \mathrm{GeV}$ between constructive and destructive interference patterns has been found for a $W^{\prime}$-boson mass in the 3-4 TeV range [21].

\section{3. $Z^{\prime}$ searches in DY channel at the LHC}

The prediction of (at least) one extra neutral vector boson is a generic feature of models where the gauge group is extended compared to the SM. Scenarios falling in that category include Grand Unified Theories (GUT), theories of dynamical electroweak symmetry breaking and extra-dimensional theories [19]. The set of models that are being specifically considered here is the same as in [20]. It consists of thirteen different models, split in three classes: GUT with an $E_{6}$ gauge group $\left(E_{6}\right)$, Generalised Left-Right symmetric models (GLR), and generalisations of the SSM benchmark scenario (GSM). No flavour changing effects are considered, and family universality of the fermion couplings is assumed. The $Z^{\prime}$ resonances are taken to be as narrow as allowed by the following commonly adopted assumption: only the direct decay to SM fermions is considered [19, and references therein]. The widths in these models are typically a few percent of the $Z^{\prime}$-boson mass, and the branching ratios (BR) to charged leptons of given flavour fall in a similar range. For the $Z^{\prime}$ search at the LHC in DY channel, we consider the process

$$
p p \rightarrow Z, \gamma, Z^{\prime} \rightarrow l^{+} l^{-} \quad l=e, \mu .
$$

The results here below summarized are described in detail in Ref. [23]. 

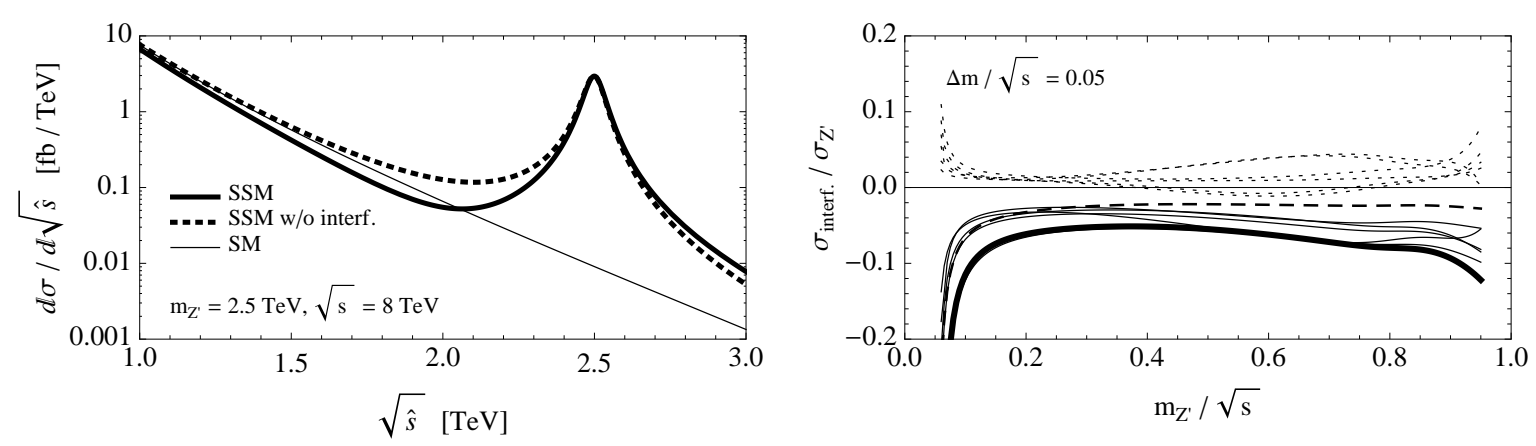

Figure 3: Left: Dilepton invariant mass distribution for the $Z^{\prime}$ SSM with interference (solid black line) and without interference (dashed black line). The SM background is given as reference by the grey solid line. Right: Relative size of interference as a function of $M_{Z^{\prime}} / \sqrt{s}$ for a fixed value of the cut $\Delta m / \sqrt{s}=5 \%$. The different lines represents all models described in Ref. [20]

The consequences of neglecting the interference between the extra $Z^{\prime}$ boson and the SM $Z$ and photon are similar to the charged case we discussed in the previous section. They are indeed sizeable, model dependent, and can reach up to O(200\%) in the SSM as can be argued from the left plot of Fig. 3. So, two possible strategies could be envisaged. Either new dedicated analyses are developed, aimed at improving the current mass bounds or at distinguishing between different $Z^{\prime}$ models, or the size of interference should be kept below the current theoretical uncertainties via appropriate cuts, so to allow a quasi-model independent analysis as in the traditional employed scheme. If opting for the latter choice, we find that the interference can be kept under control by imposing a simple but powerful kinematical cut. This is shown in the right plot of Fig. 3 where the ratio between the interference contribution to the cross section and the pure BSM signal is plotted as a function of the $Z^{\prime}$-boson mass normalized to the collider energy. There one can see that applying the following invariant mass cut, where $\sqrt{s}$ represents the collider energy,

$$
\Delta m / \sqrt{s}=\left|M_{l l}-M_{Z^{\prime}}\right| / \sqrt{s} \leq 0.05
$$

the interference effect remains below the $\mathrm{O}(10 \%)$ over almost the entire $Z^{\prime}$ mass range. In other words, the result is quasi independent on the $Z^{\prime}$ mass value, and is therefore quite robust.

\section{Conclusions}

The results presented here show that even in the presence of a single extra gauge boson, where the interference cannot be maximal, it may still become an important effect when considering the BSM cross-section in a large enough kinematic range. The result holds for both $W^{\prime}$ and $Z^{\prime}$ boson searches. The main conclusion is that the unfolded fully integrated BSM signal cross section is not an appropriate observable to present experimental results and exctract $Z^{\prime}$ and $W^{\prime}$ exclusion mass bounds, as it might not be positive definite. Moreover, neglecting the interference can bring to over/under estimate the $95 \%$ C.L. exclusion bound on the mass of new spin-1 particles by a factor of $\mathrm{O}(10-20 \%)$, if adequate cuts are not imposed. At the light of these results, novel ways of 
presenting experimental results just started, as discussed in others contributions to this Conference. But, much work is still ahead.

\section{References}

[1] L. Barzé et al., arXiv:13024606

[2] M. Dittmar, Phys. Rev. D55 (1997)

[3] T. G. Rizzo, JHEP 0705 (2007) 037.

[4] F. Petriello and S. Quackenbush, Phys. Rev. D77 115004 (2008)

[5] A. Papaefstathiou and O. Latunde-Dada, JHEP 0907 (2009) 044

[6] T. G. Rizzo, JHEP 0908, 082 (2009)

[7] Cheng-Wei Chiang et al., Phys. Rev D85 (2012) 015023.

[8] T. Aaltonen et al., Phys. Rev D83 (2011) 031102

[9] V. M. Abazov et al., Phys. Lett. B695 (2011) 88

[10] G. Aad et al. arXiv:1209.4446

[11] ATLAS-CONF-2013-017, Mar 2013

[12] S, Chatrchyan et al. Phys. lett. B720 (2013) 63; arXiv:1302.2812

[13] S Chatrchyan et al., JHEP 1208 (2012) 023

[14] G. Aad et al., arXiv:1209.2535

[15] CMS-PAS-EXO-12-060, 2013.

[16] J. C. Pati and A. Salam, Phys. Rev. D10 (1974) 275; R. N. Mohapatra et al., Phys. Rev. D11 (1975) 3319; G. Senjanovic and R. N. Mohapatra, Phys. Rev. D12 (1975) 1502.

[17] N. Arkani-Hamed, S. Dimopoulos and G. R. Dvali, Phys. Lett. B429 (1998) 263; I. Antoniadis et al., Phys. Lett. B436 (1998) 257; L. Randall and R. Sundrum, Phys. Rev. Lett. 83 (1999) 3370.

[18] G. Altarelli et al., Z Phys. C45 (1989) 109.

[19] P. Langacker:2008yv, Rev. Mod. Phys. 81 (2009) 1199.

[20] E. Accomando et al., Phys. Rev. D83 (2011) 075012.

[21] S. Chatrchyan et al., JHEP 1208 (2012) 023; CMS-PAS-EXO-12-010 (2012).

[22] E. Accomando et al., Phys. Rev. D85 (2012)115017.

[23] E. Accomando et al., arXiv:1304.6700. 